\title{
Role of different imaging modalities in the evaluation of normal and diseased thymus
}

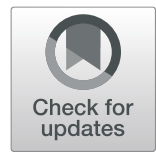

S. A. Hussein ${ }^{*}$, Y. Y. Sabri², M. A. Fouad², H. H. Al-Zawam³ and N. M. Mohamed²

\begin{abstract}
Background: The appearance of the thymus on imaging studies showed great variability. Thymus imaging is a great challenge. This cross-sectional prospective study investigated the diagnostic value of different imaging modalities in the evaluation of normal and diseased thymus. Chest X-ray was done as a primary survey. Chest computed tomography (CT) was done for 82 patients, chest magnetic resonant imaging (MRI) was done for 40 patients, and 18 flurodeoxy glucose positron emission tomography (18 FDG-PET) was done for 2 patients. Ultrasonography (US) of the anterior mediastinum was done for 2 pediatric patients. Histopathological assessment was done for all cases with diseased thymus.

Results: The study included 158 patients divided into 2 groups: group A, 79 patients with normal thymus for age and group B, 79 patients with thymic lesions detected by one or more of the used modalities. The large-sized thymus was in 79 patients, who were subcategorized to patients with myasthenia gravis (12 patients) and patients with mediastinal masses (67 patients). Imaging findings in normal and diseased thymus gland were compared and lesion analysis by various modalities displayed with histopathological correlation.

Conclusion: There are different imaging modalities for thymus gland evaluation. $\mathrm{CT}$ is beneficial for primary assessment giving the morphology and density, and MRI is the imaging modality of choice in diseased thymus owing to functional assessment of the gland; however, X-ray may be helpful as a primary survey tool before CT and MRI. Chest ultrasound could be a suitable primary assessment tool in pediatrics before MRI because it is an easy bedside test.
\end{abstract}

Keywords: Thymus gland, Imaging modalities, Histopathological correlation

\section{Background}

The thymus gland is one of the lymphatic organs that play an important role in the development and maturation of the immune system in childhood. The normal thymus is a triangular, bi-lobed organ; its shape, size and composition changes with age. The thymus is larger in infants but gradually becomes involuted throughout maturation and replaced by fat. The thymus is present in the anterior mediastinum. The thymus overlies the pericardium, aortic arch, left innominate vein, and trachea. It extends superiorly to the lower pole of the thyroid and inferiorly to the diaphragm $[1,2]$.

\footnotetext{
* Correspondence: Sabah.Hussein@kasralainy.edu.eg

${ }^{1}$ Chest Department, Faculty of Medicine, Cairo University, Cairo, Egypt

Full list of author information is available at the end of the article
}

The features of the thymus such as size, shape, attenuation on computed tomography (CT), and signal characteristics on magnetic resonance imaging (MRI) show great variability in the appearance. It is a great challenge to differentiate normal from diseased thymus due to this great variety of imaging appearances. Lesions of the thymus may be seen in some systemic disorders or as one of the manifestations of myasthenia gravis and some immunodeficiency disorders. Thymic lesions mostly appear as incidental findings on imaging for other causes, and thymus imaging is a great challenge [3].

On frontal chest radiographs, the pediatric thymus gland is usually visible up to the 2nd year of age or beyond but invisible in adult age and beyond [4]. In young children, ultrasonography is a suitable procedure for 
thymic diagnosis. It helps to identify the nature of a suspected thymic mass either solid or cystic and to detect the presence of fat or calcification within. It also helps in image-guided aspiration or biopsies of thymic lesions. The thymus is seen on computed tomography of the chest as a quadrilateral-shaped soft tissue density structure in the anterior mediastinum with convex margins. It becomes triangular in shape with straight or concave margins as age increases. Thymus density on computed tomography (CT) decreases with age in children (from 80 to 56 Hounsfield unit (HU)) due to fatty replacement [5].

On MRI, the signal intensity of the thymus gland appears homogeneous and is greater than that of the muscle on T1-weighted images and of intermediate signal intensity on T2-weighted images [2]. Fat saturation does not decrease the signal intensity of the thymus; however, chemical shift imaging has been used to assess the fatty replacement of the thymus and identify infiltrative disorders so with diffusion-weighted images, MRI can help in differentiating the thymus hyperplasia from thymic malignancy [6].

On PET scans, normal thymus may be barely visible. However, it may show striking FDG avidity in rebound hyperplasia, causing false alarm for recurrent lymphoma [2].

This study assessed the role of different imaging modalities in the comprehensive evaluation of normal and diseased thymus and the differentiation between thymic hyperplasia and thymic malignancy with different imaging modalities and histopathological correlation.

\section{Methods}

The present study is a prospective study which included 158 patients. Males were 96 and females were 62; their age range was from 2 months to 70 years with age average of about 26 years. Patients were recruited from chest, radiology, pediatrics, oncology, and neurology departments in Kasr Al-Ainy hospitals, in the period from July 2017 till December 2017. The study has been approved by the human research ethics committee, Faculty of Medicine, Cairo University, and a written informed consent was obtained from all patients or their parents in pediatric cases.

Patients with thymic disease in different imaging modalities and patients of different age groups with normal thymus are included in this study. Exclusion from the study was depending on the modality used in studying the thymus. In X-ray and $\mathrm{CT}$, the main contraindication was pregnancy and the main MRI contraindication was claustrophobia and cases with metallic MRI incompatible cardiac pacemakers, cochlear implants, or certain cardiac valves and stents. Also, patients with contraindication for biopsy from the diseased thymus were excluded such as patients with a bleeding tendency or major organ failure.

Patients underwent different imaging modalities according to the clinical condition; chest X-ray was done as a primary survey. CT chest was done for 82 patients, MRI chest was done for 40 patients, and 2 patients underwent 18 FDG positron emission tomography (PET/CT). Ultrasonography of the anterior mediastinum using a high-frequency probe in longitudinal and transverse scan was done for 2 pediatric patients. For all cases with diseased thymus especially thymic neoplasm, tissue biopsy was obtained using either open thoracotomy, US, or CT-guided biopsies for histopathological confirmation and histopathological correlation with the provisional diagnosis of the used imaging modality.

Multi-detector computed tomography (MDCT) of the chest was performed using 16-multislice GE bright speed CT scanner with the following parameters: helical acquisition, $120 \mathrm{kV}, 25 \mathrm{~mA}$; helical thickness of $1.25 \mathrm{~mm}, 1$ $\mathrm{mm}$ interval; FOV of $351 \mathrm{~mm}$ down from the level of the renal arteries up to the root of the neck; and total exposure time of $0.8 \mathrm{~s}$ during a breath hold. Different plans were obtained including mediastinal window and lung window and axial images with coronal and sagittal reconstruction.

MRI technique was done by a 1.5 - $\mathrm{T}$ unit (Philips Achiva) with a dedicated torso phased-array surface coil. MRI sequences axial T1WI, T2WI, STIR, coronal T2WI, sagittal T1WI, DWI (diffusion-weighted images) and ADC (apparent diffusion coefficient) map with ADC value $(b$ values $0,500,1000)$ and transverse gradientecho T1-weighted in- and opposed-phase imaging. Imaging parameters were as follows: $275-400-\mathrm{mm}$ field of view, 5-mm section thickness, $0.5-\mathrm{mm}$ intersection gap, flip angle of $90^{\circ}$, repetition time of $154 \mathrm{~ms}$, and in-phase and opposed-phase echo times of 4.6 and $2.3 \mathrm{~ms}$, respectively. An anterior-to-posterior phase-encoding direction was used on the in-phase and opposed-phase images. Diffusion-weighted images and ADC map were also obtained for the evaluation of the thymic masses.

\section{Analysis of MR image}

MRI images were assessed qualitatively and quantitatively. For qualitative assessment, the drop-off of the thymic signal in out-of-phase relative to in-phase images was assessed. In the quantitative assessment, signal intensity measurements within the thymus and para-spinal muscle were obtained with an electronic cursor by using regions of interest (ROI) of the same size that was placed over the same positions within the normal, hyperplastic thymus or tumor or para-spinal muscle on both in-phase and opposed-phase images in each case by the three readers, independently. The selection of the ROI placement was first made on the opposed-phase image, 
to avoid the inclusion of areas of signal void at the interfaces between the fat-dominant and water-dominant tissues (India ink artifact), and then the position was mirrored so it appeared on the in-phase image in the exact same location. On the opposed-phase T1-weighted image, the readers placed the ROI on the anterior mediastinal soft tissue that exhibited the highest signal intensity, avoiding cystic, necrotic, or calcified components. The ROI over the para-spinal muscle was positioned without including obvious fat striations whenever possible. In the following equation, the chemical shift ratio (CSR) was determined by comparing the signal intensity of the thymus gland (tSI) with that of the paraspinal muscle (mSI) on both in-phase (in) and opposed-phase (op) images: $\mathrm{CSR}=(\mathrm{tSI} I \mathrm{p} / \mathrm{mSIop}) /(\mathrm{tSIin} / \mathrm{mSIin})$.

\section{Results}

This study included 158 patients (96 males and 62 females). Age ranged from 2 months to 70 years with age average about 26 years. The main presenting symptoms were respiratory complaints mainly dyspnea and cough in about $122(77.2 \%)$ patients, muscle weakness in about 17 (10.8\%) patients, collagen vascular disease mainly dermatomyositis and polymyositis in about 14 (8.9\%) patients, and incidental findings of thymic lesion on imaging of preoperative assessment in about 5 (3.1\%)

Patients were assigned to two main groups:

- Group A: patients with normal thymus for age.

- Group B: patients with thymic lesions detected by one or more of the imaging modalities used in this study.

\section{Group A}

Group A included 79 patients with normal thymus detected in 53 males and 26 females; their ages ranged from 2 months to 70 years old with an average age of about 14 years old. Normal thymus could be detected in those patients by using different modalities: X-ray in $41 /$ 79 (53\%), CT in 36/79 (46\%), and US in 2/79 (1\%).

\section{Plain chest X-ray (CXR)}

CXR with normal thymus could be detected in 41 patients, 29 males, and 12 females with an age range from
2 months to 45 years with an average of about 6 years old. Patients with normal thymus according to the age were $18 / 79$ patients in the age $<2$ years, $13 / 79$ patients in the age $2-5$ years, $3 / 79$ patients in the age $5-10$ years, $5 / 79$ patients in the age $10-20$ years, and $2 / 79$ patients in the age group $>20$ years. The thymus is seen as a superior mediastinal widening. Borders of normal thymus were either convex, straight, wavy, or sail-like. The commonest thymic border was the wavy border which was presented in about $50 \%$ of patients.

\section{CT chest}

CT chest was informative for 36 patients, 22 males and 14 females with an age range from 5 months to 67 years with the average age of about 25 years. In all cases, the thymus is seen as a mass of homogenous density in the anterior superior mediastinum iso-dense to the chest wall muscles. The size (side-to-side dimensions), shape, homogeneity, and density of the thymus differ with age (Table 1).

\section{Ultrasonography}

Ultrasonography was done for two patients $(2$ males, their ages were 6 and 9 months). Thymus appears with echo texture resembling that of the spleen or liver in being homogeneous and coarsely reticular, while its echogenicity is slightly lower than that of the thyroid gland.

\section{Group B}

Group B included 79 patients with thymic lesions detected by one or more of the modalities used in this study. There are 43 males and 36 females, and their ages ranged from 1 to 70 years old with an average age of about 42 years old. Large-sized thymus cases were subcategorized according to their main presentation to patients presenting with myasthenia gravis symptoms (12/ 79 cases, $15.19 \%)$ and patients presenting with mediastinal masses (67/79 cases, $84.81 \%)$.

\section{Patients presenting with myasthenia gravis $(n=12)$}

This group included 6/12 cases histopathologically diagnosed as thymolipoma, 3/12 cases diagnosed as thymic hyperplasia, and 3/12 cases diagnosed thymoma. Regarding imaging findings of thymolipoma cases (Fig. 1), on

Table 1 Different sizes, shapes, and density of thymus gland in CT chest mediastinal window according to age

\begin{tabular}{|c|c|c|c|c|c|c|c|c|}
\hline \multirow[t]{2}{*}{ Age } & \multirow[t]{2}{*}{ No. } & \multicolumn{3}{|c|}{ According to shape } & \multicolumn{3}{|c|}{ According to density } & \multirow{2}{*}{$\begin{array}{l}\text { Size range } \\
\text { (average) }\end{array}$} \\
\hline & & $\begin{array}{l}\text { Both borders } \\
\text { are convex }\end{array}$ & $\begin{array}{l}\text { One border convex } \\
\text { and one straight }\end{array}$ & $\begin{array}{l}\text { Both borders } \\
\text { are straight }\end{array}$ & $\begin{array}{l}\text { Homogenous soft } \\
\text { tissue density }\end{array}$ & $\begin{array}{l}\text { Heterogeneous soft } \\
\text { tissue and fat density }\end{array}$ & Fatty replacement & \\
\hline$<10$ years & 16 & 5 & 3 & 8 & 16 & - & - & $2-5.5(3)$ \\
\hline $10-20$ years & 4 & - & - & 4 & 2 & 2 & - & $1.5-3.5(2.3)$ \\
\hline$>20$ years & 16 & - & - & 16 & - & - & 16 & $1.3-2.5(1.5)$ \\
\hline
\end{tabular}

No. total number of cases, - no cases 

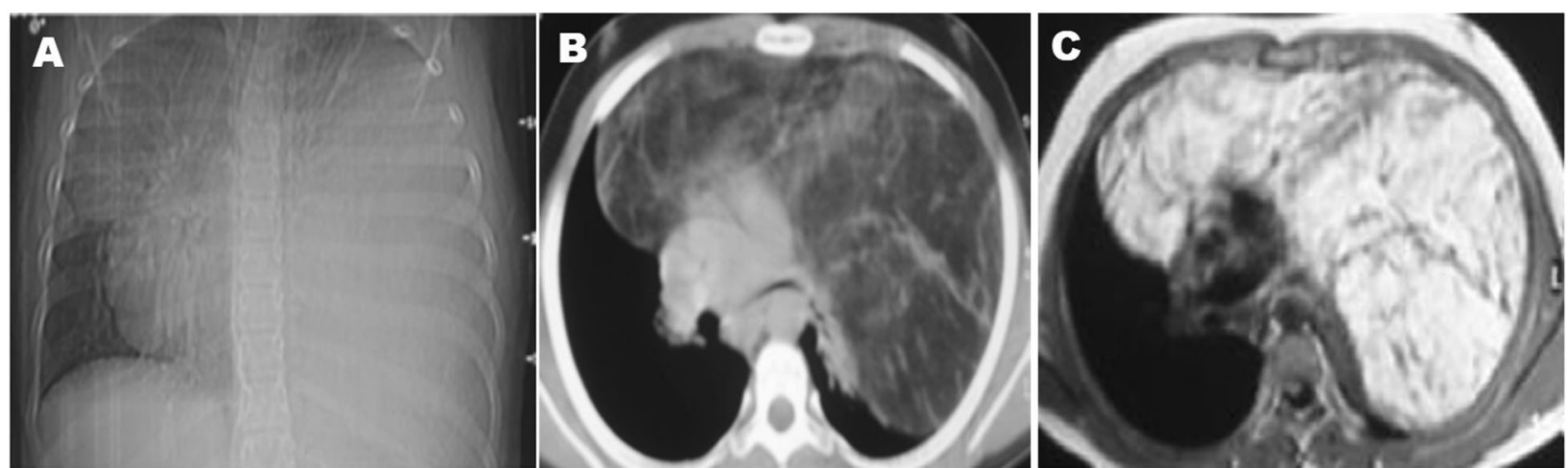

Fig. 1 a Chest X-ray PA view shows a near-total opacification of the left hemithorax and right upper lung zone merging with the mediastinum. $\mathbf{b}$ Axial CT chest mediastinal window shows a large fat attenuating mass with thin hypodense fibrous septae. c MRI lung T1 WI shows the hyperintense signal of the mass confirming its fatty nature. Imaging diagnosis and histopathologic assessment revealed "thymolipoma"

plain chest X-ray, 1 case showed marked left-sided diffuse mediastinal widening reaching to the diaphragm without silhouetting it, a sign that suggests its fat content. Histopathological assessment proved that it was thymolipoma. CT chest revealed a large fatty anterior mediastinal mass containing a small amount of soft tissue in 4 cases that were also pathologically proven to be thymolipoma. MRI revealed in 2 cases, the anterior mediastinal mass with fat signals is high in $\mathrm{T} 1$ and $\mathrm{T} 2$ with fibrous septae. Regarding thymic hyperplasia cases, CT chest revealed diffuse, symmetric thymic enlargement with a smooth contour, interspersed fat and soft-tissue elements, normal vessels, and preserved adjacent fat planes in 2 cases (pathologically proven to be thymic hyperplasia) and MRI showed in 1 case of the enlarged thymus with preserved smooth contour with a signal drop in out-phase images compared to the in-phase images $\operatorname{CSR}=0.92$, free diffusion. Regarding thymoma cases, the imaging modality used in the 3 cases for provisional diagnosis was MRI, which revealed illdefined anterior mediastinal mass with low signal on T1 and high or mixed signal on T2 and restricted diffusion, CSR $>1$ (no signs of signal drop)

\section{Patients presented with mediastinal mass (with no symptoms of myasthenia gravis patients; $n=67$ )}

All these patients presented with dyspnea and dry cough. Plain CXR was showed irregular mediastinal widening for 8 patients. CT chest was diagnostic for 39 patients. Thymic hyperplasia was diagnosed in 18 patients, thymoma in 12 cases, lymphoma in 4 cases, thymic cyst in 1 case (Fig. 2), and invasive thymoma in 4 cases. MRI chest was conclusive in 29 patients with anterior mediastinal mass. Thymoma was diagnosed in 10 cases, lymphoma in 5 cases, thymus hyperplasia in 10 cases, teratodermoid tumor in 1 case, and thymic carcinomain 2 cases. The diffusion-weighted images showed facilitated diffusion in patients with thymus hyperplasia compared to restricted diffusion in cases of thymic tumors. CSR in thymic hyperplasia was $0.37 \pm 0.15$ with a drop of signal in out-of-phase images (Fig. 3) and in thymic tumor group ranging from $0.9-1.8$, with no drop of signal in out-of-phase images (Fig. 4), except in a case of teratodermoid tumor, where it showed some areas of suppressed signal in out-of-phase images (Fig. 5). PET-CT was done for 2 male patients with lymphoma showing an increase in the FDG uptake of the thymus and other lymph nodes.

\section{The spectrum of histopathological diagnoses of the different thymic lesions in group $B$ patients who had large- sized thymus included (Fig. 6)}

- Thymic hyperplasia (31/79, 39.24\%), 20 (64.52\%) of them were provisionally diagnosed by CT chest while 11 (35.48\%) of them were diagnosed using chest MRI.

- Thymoma (25/79, 31.65\%), 12 (48\%) of them were diagnosed using CT chest and 13 (52\%) diagnosed using an MRI chest.

- Invasive thymoma (4/79, 5.06\%), all were provisionally diagnosed using CT chest.

- Thymic carcinoma (2/79, 2.53\%), all were provisionally diagnosed using MRI chest.

- Lymphoma $(9 / 79,11.39 \%), 4$ (44.44\%) of them were diagnosed by CT chest while the other 5 (55.56\%) cases were diagnosed using MRI.

- Thymolipoma (6/79, 7.59\%), 4 of them were diagnosed by CT chest and 2 were diagnosed using MRI.

- Thymic cyst (1/79, 1.27\%), diagnosed by chest CT.

- Terato-dermoid tumor $(1 / 79,1.27 \%)$, diagnosed using an MRI chest. 

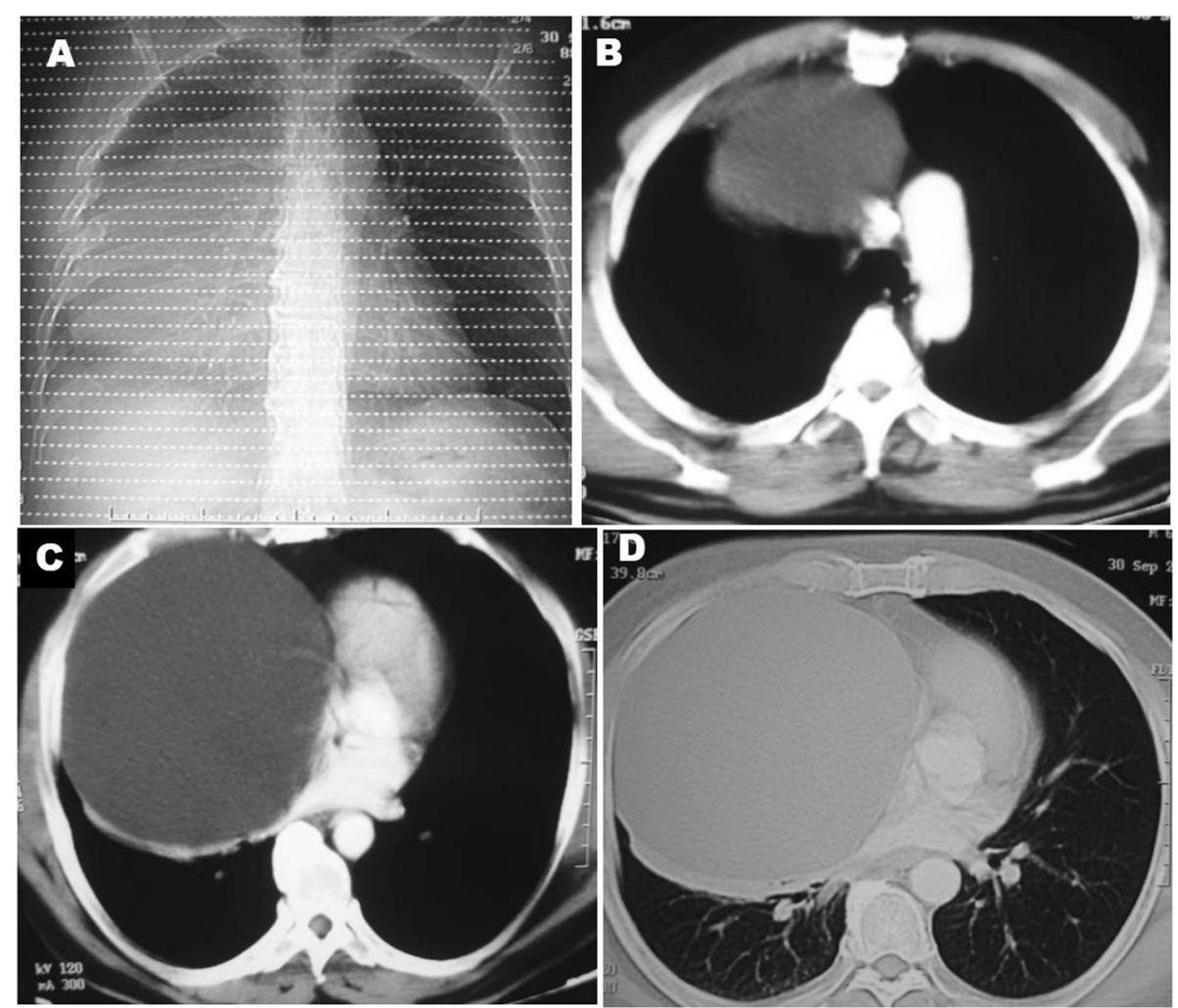

Fig. 2 Sixty-year-old male patient complaining of dyspnea. a Reconstruction image showing right-sided opacity not silhouetting the cardiac border. $\mathbf{b}-\mathbf{d}$ Contrast-enhanced axial CT chest mediastinal and lung windows showing anterior mediastinal fluid attenuating non-enhancing cystic lesion. It shows a thin rim with no enhancement. CT diagnosis and histopathological confirmation revealed "thymic cyst"

So, in group B cases, CT was conclusive in $45 / 79$ cases (56.96\%) and MRI in 34/79 cases (34.04\%) (Fig. 6).

\section{Discussion}

The thymus gland evaluation poses a challenge due to age-related changes regarding thymic size, shape, and tissue composition [7]. Multiple disorders can affect the thymus such as hyperplasia, cysts, and neoplasms including benign and malignant tumors [8]. Multiple noninvasive imaging modalities can be used for the evaluation of the thymus such as ultrasonography, computed tomography. (CT), magnetic resonance imaging. (MRI), and nuclear scintigraphy (including PET CT). They can provide unique and often complementary information in the evaluation of normal and thymic disorders [3].

In this study, 158 patients were included (96 male and 62 female), and their age range was from 2 months to 70

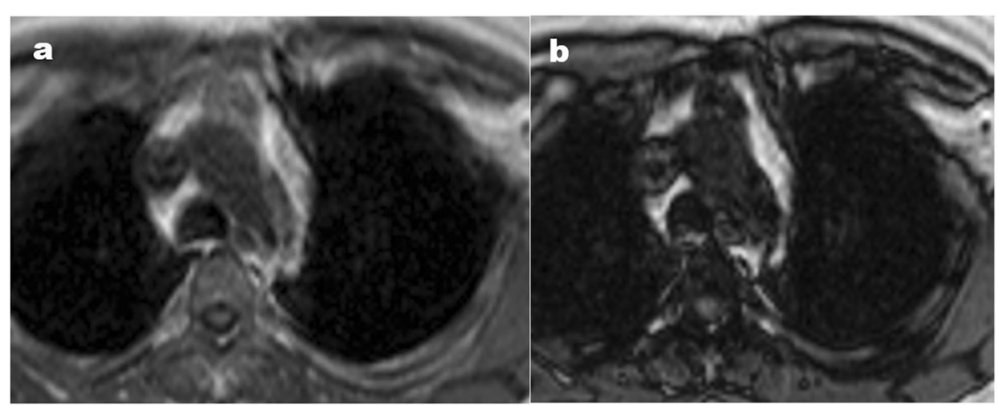

Fig. 3 Axial MRI chest in a 28-year-old male revealed anterior mediastinal mass that is iso-intense to muscle on in-phase images (a) and $\mathbf{b}$ demonstrating a decrease in signal intensity on opposed-phase images indicating microscopic fat, CSR $=0.4$. These findings are compatible with thymic hyperplasia. MRI diagnosis and histopathological confirmation revealed "thymic hyperplasia" 

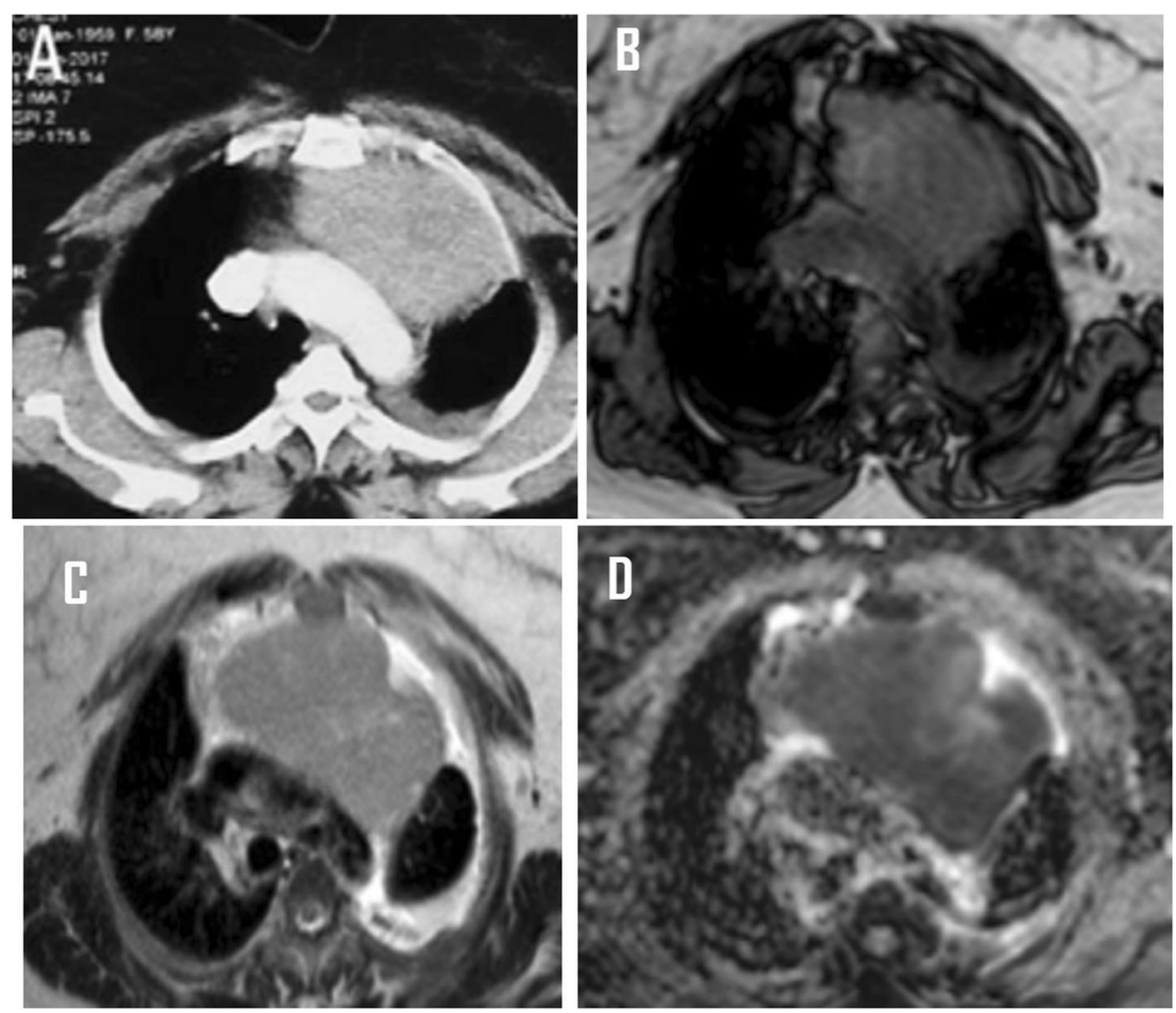

Fig. 4 Fifty-eight-year-old female presenting with cough and dyspnea. a Axial contrast-enhanced CT chest mediastinal window shows a welldefined homogenously enhancing anterior mediastinal soft tissue mass. MRI was done, and axial chemical shift sequences show no suppression on the out-phase (b) compared to the in-phase images (c) with CSR of 1.13. In diffusion-weighted images, ACD map (d) shows restricted diffusion with $\mathrm{ADC}$ value $=0.9$. Histopathological assessment revealed thymoma

years with an average age of about 26 years. They underwent different imaging modalities according to their condition. Accordingly, they subdivided into 2 groups: patients with normal thymus for age (group A) and patients with diseased thymus detected by one or more of the modalities used (group B).

In our study, the patients with normal thymus appeared in CXR as superior mediastinal widening with sharp outline ranging from laterally convex, straight to wavy, or with sail-like border. The wavy outline was the most common one which was seen in about $50 \%$ of the patients while the sail-like outline was the least one which appeared in only 2 patients (2\%) matching with Nasserie et al.'s results that stated that sail-like outline is seen only in $3-15 \%$ of cases [2].

In those patients with normal CT appearance of the thymus, the thymus appeared as an anterior superior mediastinal structure with well-defined borders which was either convex or straight unilaterally or bilaterally. The majority of the patients in our study were with bilateral straight borders seen especially with an increase in age above 10 years giving the thymus its triangular appearance that matches with Manchanda et al. who reported that the thymus in children less than 5 years old appears usually with biconvex borders and with age, the thymus becomes triangular with straight or even with concave borders [5].

The size of the thymus also varies with age, and sideto-side diameter was measured in normal thymus using CT in different age groups with an average $3 \mathrm{~cm}$ in children less than 10 years old and decreased until $1.5 \mathrm{~cm}$ in patients more than 20 years old with fatty infiltration as it could not be seen after 35 years. The density of the thymus is also observed to decrease with age; it appeared of homogenous soft tissue density in patients less than 10 years old and gradually became heterogeneous in fat and soft tissue densities representing residual thymic tissue and complete fatty infiltration noted in patients above 35 years [8].

Araki et al.'s extended study on 2540 participants of the normal thymus gland divided the normal gland according to CT attenuation as follows: score 0 , complete fatty replacement with no identifiable soft tissue density in the thymic bed which was in $74 \%$ of cases; score 1 , predominantly fatty thymus which was in $18 \%$ of cases; score 2 , one-half fatty and one-half soft tissue attenuated thymus which was in 7\% of cases; and score 3, predominantly soft tissue attenuated thymus which was in $1 \%$ of 

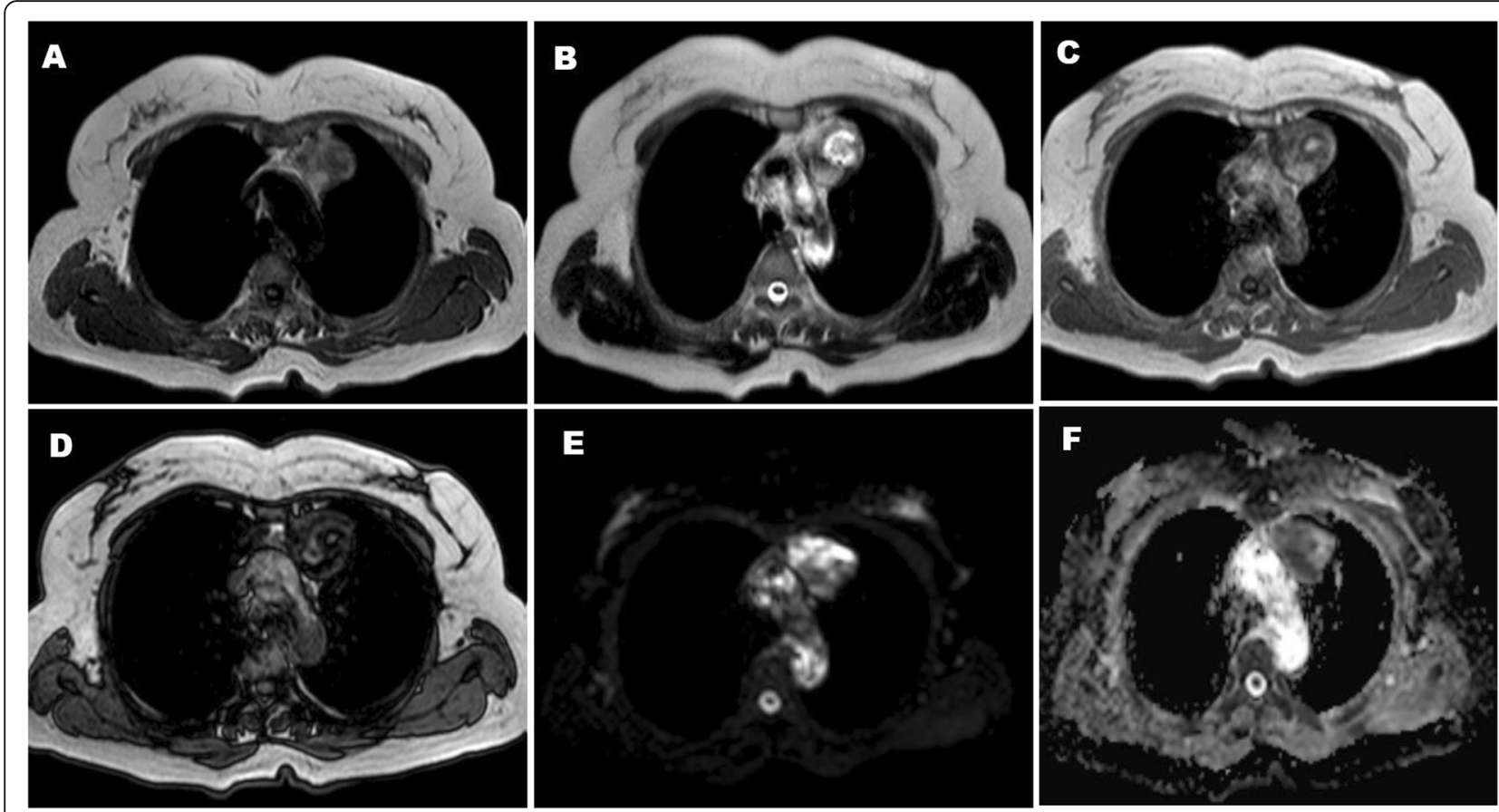

Fig. $5 \mathrm{MRI}$ chest in a 45-year-old female patient shows a rather well-defined superior anterior mediastinal lobulated soft tissue lesion eliciting heterogenous low $\mathrm{T1}$ (a) and mixed signal in T2WI signals (b), with areas of restricted diffusion in out-of-phase image (d) compared to in-phase $(\mathbf{b}, \mathbf{c})$ image. It elicits diffusion restriction $(\mathbf{e}, \mathbf{f})$ with $A D C$ value $=0.8$. Histopathological confirmation revealed terato-dermoid tumor

cases [9]. Our normal control cases ranged from score 0 in $86.7 \%$ of cases and score 1 in $13.3 \%$ of cases.

Mean CT attenuation in our cases was - $49 \mathrm{HU}$, which is more or less the same as Araki et al., which was - 39.3 HU in females and - 51.7 HU in males. The thymus morphology that was most frequently seen was the pyramidal thymus with a straight margin in $71 \%$, followed by pyramidal thymus with convex margin in
$22 \%$, pyramidal with concave margin in $4 \%$, and irregular in $3 \%$ [9]. In our study, wavy borders were seen in $50 \%$, straight borders in $33 \%$, and convex borders in $15 \%$ of cases.

Mean thymic measurements in AP diameters were $16-26 \mathrm{~mm}$ in female and $25-31 \mathrm{~mm}$ in male and transverse diameters $21-30 \mathrm{~mm}$ in female and $32-37 \mathrm{~mm}$ in male [9]; in this study, the mean side-to-side dimension

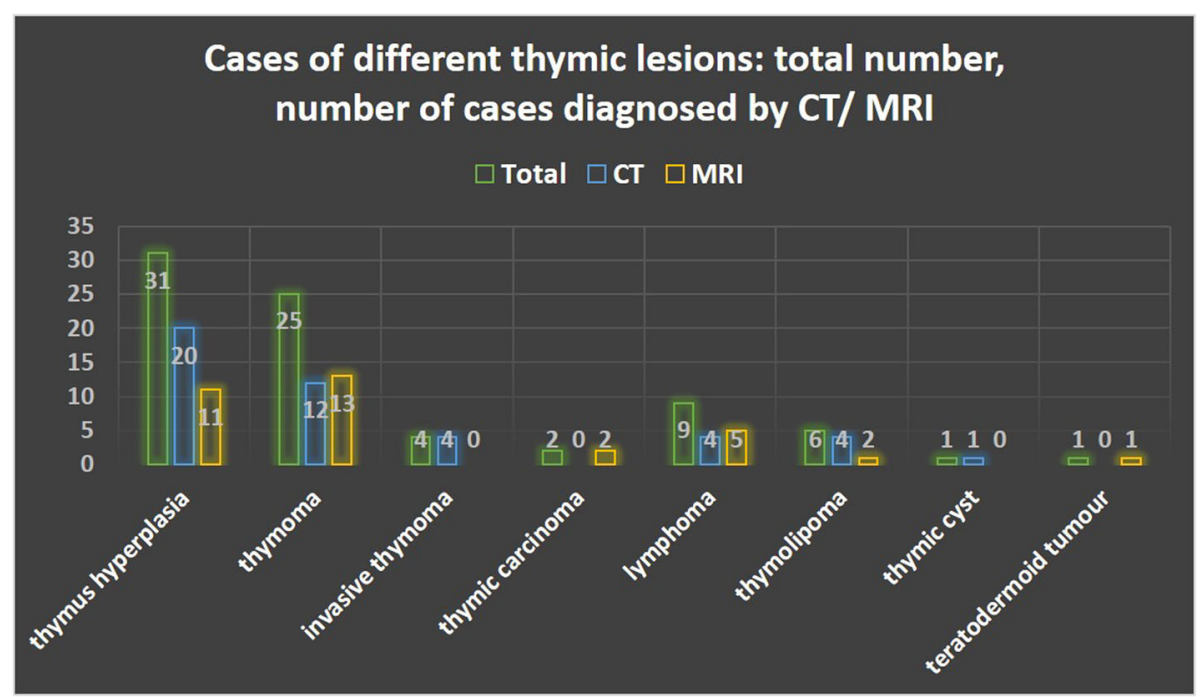

Fig. 6 The histopathological results of cases with different thymic lesions: total number, number of cases diagnosed by CT/MRI 
in normal subjects was $13-25 \mathrm{~mm}$ and $7-16 \mathrm{~mm}$ for gland thickness.

By US, in the two patients who underwent ultrasound for thymus, it appeared with echo texture being homogeneous and coarsely reticular resembling that of the spleen and liver, while its echogenicity was slightly lower than that of the thyroid gland matching with Nasserie et al. who mentioned that the study which was done on 56 infants (0-10 months) showed that the echogenicity of the thymus was similar to that of the liver and spleen in 42 patients (75\%), and in 14 infants, it was slightly less homogenous than liver [2].

In our study, 17 patients were diagnosed with myasthenia gravis and underwent various imaging modalities; 5 of them had normal thymus in X-ray and CT. One case had a mediastinal mass by CXR (histologically proven to be thymolipoma), 6 patients underwent CT chest (2 diagnosed pathologically as thymic hyperplasia and 4 as thymolipoma). MRI was done for 5 patients (3 cases were diagnosed pathologically as thymoma, 1 case as thymic hyperplasia, and 2 cases as asthymolipoma). So, about $30 \%$ of our patients who were diagnosed with myasthenia were normal, $18 \%$ with hyperplasia, $18 \%$ with thymoma, and $34 \%$ with thymolipoma. These findings were comparable to Strollo et al. that stated that thymic hyperplasia is seen in $65-85 \%$ of patients with myasthenia, $10-15 \%$ of myasthenia gravis patients have thymoma, and $20 \%$ have normal or involuted thymus [10].

In this study, an enlarged thymus appearing as anterior mediastinal mass as the main finding was present in 67 patients complaining mainly of dyspnea. Histopathological assessment of those patients could detect different thymic pathologies as thymoma in $31.65 \%$, thymic hyperplasia in $39.24 \%$, lymphoma in $11.39 \%$, thymolipoma in $7.59 \%$, invasive thymoma in $5.06 \%$, thymic carcinoma in $2.53 \%$, thymic cyst in $1.27 \%$, and terato-dermoid tumor in $1.27 \%$. It is difficult to show the difference between the normal and the hyperplastic thymus gland as both might have a variable amount of fat tissue. The main differentiating feature is the shape which is mostly pyramidal with a straight margin in the normal thymus, although a hyperplastic thymus may be suggested by convex margin [9]

This study demonstrated that thymic hyperplasia could be suggested by $\mathrm{CT}$ which showed the gland of true thymic hyperplasia shows symmetric enlargement with preservation of its normal bi-lobed shape and no discrete mass. But occasionally, the gland may have a convex margin and appear oval. The gland had smooth contour, normal vessels, and preserved surrounding fat planes while thymoma appeared in the anterior mediastinum as a lobulated, well-defined, soft tissue density mass with rounded margins.

Thymic lymphoid hyperplasia can appear on CT scan as anterior mediastinal mass (20\%), as diffusely enlarged thymic gland (35\%), or as normal thymus (45\%). The enlarged thymus borders are most often concave [2].

MRI was helpful in the differentiation between normal and hyperplastic thymus from thymic tumors by using chemical shift ratio (CSR) [9]. CSR is calculated to assess the presence of microscopic fat and tissue within the same voxel [5]. Unlike the thymic tumors, MRI of hyperplastic thymus showed a drop of signal intensity in outof-phase images compared to the in-phase images indicating the presence of microscopic fat. CSR less than 0.9 was detected in all patients with thymic hyperplasia while those of tumors were more than 0.9. The results were comparable to the study done by Inaoka et al. who demonstrated a mean CSR (standard deviation) of 0.614 _ 0.130 in patients with thymic hyperplasia versus 1.026 _ 0.039 in patients with thymic epithelial tumors [9]. The diffusion-weighted images showed facilitated diffusion in patients with thymus hyperplasia compared to restricted diffusion in cases of thymic tumors.

PET-CT which was done for 2 patients with lymphoma showed an increase in the FDG uptake at the thymus and other lymph nodes with a standard uptake value (SUVmax) of 10.4 and 8.2 which matches with Mittal et al. who reported that PET CT is helpful in the diagnosis of lymphomatous infiltration of the thymus as it shows an increased uptake of FDG and also helps in detecting other nodal affection with SUVmax $>3.4$ in cases of lymphoma [1].

CT was the modality of choice in the primary assessment of thymic lesion in adult cases; however, MRI mediastinum and PET-CT have a growing role [11]. Chest US is the modality of choice in primary assessment in pediatric cases [5].

\section{Conclusion}

At imaging, anatomical variations and dynamic changes of thymus found to be the main source of confusion with pathological conditions. Using the different radiological modalities can help in differentiating the normal thymus from the benign and malignant thymic diseases and avoiding mismanagement. There are different imaging modalities for thymus gland evaluation. CT is beneficial for primary assessment giving the morphology and density, and MRI is the imaging modality of choice in diseased thymus owing to functional assessment of the gland; however, X-ray may be helpful as a primary survey tool before CT and MRI. Chest ultrasound could be a suitable tool for primary assessment in the pediatric age group before MRI if needed because it is an easy bedside test.

\section{Abbreviations}

CT: Computed tomography; MRI: Magnetic resonance imaging; 18 FDGPET: 18 flurodeoxy glucose positron emission tomography; US: Ultrasonography; HU: Hounsfield unit; MDCT: Multi-detector computed tomography; DWI: Diffusion-weighted images; ADC: Apparent diffusion 
coefficient; ROI: Regions of interest; CSR: Chemical shift ratio; tSI: Signal intensity of the thymus gland; SUV: Standard uptake value

\section{Acknowledgements}

The authors thank all patients for the use of their data in this research. The authors also thank our colleagues in the chest, radiology, and oncology departments, Cairo University, for their assistance.

\section{Authors' contributions}

All authors have been contributed actively in the work and the manuscript has been read and approved by all the authors for submission. SA Hussein: study conception or design, data acquisition and analysis, interpretation of data, manuscript drafting, revision, and final approval. YY Sabri: study conception or design, interpretation of radiologic images, manuscript drafting, revision, and final approval. MA Fouad: study conception or design, data analysis and interpretation, manuscript drafting, revision, and final approval. HH Al-Zawam: manuscript drafting, revision, and final approval. NM Mohamed: Study conception or design, data acquisition, manuscript drafting, revision, and final approval.

\section{Funding}

This research did not receive any specific grant from funding agencies in the public, commercial, or not-for-profit sectors.

\section{Availability of data and materials}

The data used during the current study are available from the corresponding author on reasonable request.

\section{Ethics approval and consent to participate}

The research ethics committee of chest department, Kasr Al Ainy, Cairo University, has approved this study, but the reference number is not applicable and not available. Written informed consent was obtained from all study participants before enrollment in the study.

\section{Consent for publication}

A written informed consent was obtained from the patients for the publication of their details.

\section{Competing interests}

The authors declare no financial or non-financial competing interests.

\section{Author details}

${ }^{1}$ Chest Department, Faculty of Medicine, Cairo University, Cairo, Egypt. ${ }^{2}$ Department of Diagnostic and Interventional Radiology, Faculty of Medicine, Cairo University, Cairo, Egypt. ${ }^{3}$ Oncology Department, Faculty of Medicine, Cairo University, Cairo, Egypt.

Received: 27 March 2020 Accepted: 8 April 2020

Published online: 10 June 2020

\section{References}

1. Mittal MK, Sureka B, Sinha M, Mittal A, Thukral BB (2013) Thymic masses: a radiological review. South African Journal of Radiology. 17(3):108

2. Nasseri F, Eftekhari F (2010) Clinical and radiologic review of the normal and abnormal thymus: pearls and pitfalls. Radiographics. 30(2):413-428

3. Bogot NR, Quint LE (2005) Imaging of thymic disorders. Cancer Imaging. 5(1):139-149

4. Goldstein AJ, Oliva I, Honarpisheh H, Rubinowitz AA (2015) Tour of the thymus: a review of thymic lesions with radiologic and pathologic correlation. CanadianAssociation of Radiologists Journal. Elsevier Inc. 66(1):5-15

5. Manchanda S, Bhalla AS, Jana M, Gupta AK (2017) Imaging of the pediatric thymus: clinicoradiologic approach. World J Clin Pediatr. 6(1):10-23

6. Inaoka T, Takahashi K, Mineta M, Yamada T, Shuke N, Okizaki A et al (2007) Thymic hyperplasia and thymus gland tumors: differentiation with chemical shift MR imaging. Radiology. 243:869-876

7. Rezzani R, Nardo L, Favero G, Peroni M, Rodella LF (2014) Thymus and aging: morphological, radiological, and functional overview. Age (Dordr). 36(1):313-351

8. Nishino M, Ashiku SK, Kocher ON, Thurer RL, Boiselle PM, Hatabu H (2006) The thymus: a comprehensive review. RadioGraphics. 26:335-348
9. Araki T, Nishino M, Gao W, Dupuis MSJ, Hunninghake GM, Murakami T et al (2016) Normal thymus in adults: appearance on CT and associations with age, sex. BMl and smoking. Eur Radiol. 26(1):15-24

10. Strollo DC (1997) Rosado De Christenson ML, Jett JR. Primary mediastinal tumors. Part 1: Tumors of the anterior mediastinum. Chest. 112(2):511-522

11. Marom EM (2010) Imaging Thymoma. Journal of Thoracic Oncology. 5(10): S296-\$303

\section{Publisher's Note}

Springer Nature remains neutral with regard to jurisdictional claims in published maps and institutional affiliations.

\section{Submit your manuscript to a SpringerOpen ${ }^{\circ}$ journal and benefit from:}

- Convenient online submission

- Rigorous peer review

- Open access: articles freely available online

- High visibility within the field

- Retaining the copyright to your article

Submit your next manuscript at $\boldsymbol{\nabla}$ springeropen.com 